\title{
Functional Diversity of Anti-Lipopolysaccharide Factor Isoforms in Shrimp and Their Characters Related to Antiviral Activity
}

\section{Shihao Li ${ }^{1,2}$, Shuyue Guo ${ }^{1,3}$, Fuhua Li ${ }^{1,2, *}$ and Jianhai Xiang ${ }^{1}$}

1 Key Laboratory of Experimental Marine Biology, Institute of Oceanology, Chinese Academy of Sciences, 7 Nanhai Road, Qingdao 266071, China; E-Mails: lishihao@qdio.ac.cn (S.L.); guoshuyue@126.com (S.G.); jhxiang@qdio.ac.cn (J.X.)

2 National \& Local Joint Engineering Laboratory of Ecological Mariculture, 7 Nanhai Road, Qingdao 266071, China

3 Graduate University of Chinese Academy of Sciences, Beijing 100049, China

* Authors to whom correspondence should be addressed; E-Mail: fhli@qdio.ac.cn; Tel.: +86-532-8289-8836; Fax: +86-532-8289-8578.

Academic Editor: Kellie L. Tuck

Received: 31 March 2015 / Accepted: 20 April 2015 / Published: 27 April 2015

\begin{abstract}
Anti-lipopolysaccharide factor (ALF) is a small protein with broad-spectrum antimicrobial activity, which has potential application in the disease control. Previously, we isolated seven ALF isoforms from the Chinese shrimp Fenneropenaeus chinensis. In the present study, their distributions in tissues of shrimp were analyzed and the data showed that different isoforms had different expression profiles, which suggested that they might have different functions. Then, the functions of different isoforms were studied by analyzing the antibacterial and antiviral activities of the functional domain of ALFs, the LPS-binding domain (LBD), which were synthesized by chemical methods. Different ALFs showed distinct antibacterial and antiviral activities, which were consistent with their diverse tissue distribution patterns. Sequence analysis on the LBD domain of different isoforms revealed that an identical lysine residue site was specifically conserved in peptides with anti-WSSV activity. In order to confirm whether this lysine residue is critical to the antiviral activity of the peptide, new peptides were synthesized by changing residues at this site. Changing the lysine residue at the specific site to other amino acid residue, the antiviral activity of the peptide apparently decreased. While replacing other residue with a lysine residue at this site in LBD peptide without anti-WSSV activity, the peptide will obtain the antiviral activity to WSSV. These results not only showed us a comprehensive
\end{abstract}


understanding on the function of ALFs from $F$. chinensis, but also provided clues for the development of ALFs as potential therapeutic drugs to WSSV.

Keywords: anti-lipopolysaccharide factor; LPS-binding domain; antibacterial; antiviral; lysine residue

\section{Introduction}

Anti-lipopolysaccharide factor (ALF) is a small protein with broad-spectrum antimicrobial activity, which has potential application in the disease control of shrimp. In the early 1980s, ALF was first isolated from hemocytes of horseshoe crab with the ability to bind and neutralize lipopolysaccharide (LPS) [1,2]. Until now, ALFs have been identified and characterized in different species of crustaceans.

ALFs have been verified responsive to different pathogen challenges and with multiple antimicrobial activities. $L v A L F$ from Litopenaeus vannamei, PmALF from Penaeus monodon, EsALF from Eriocheir sinensis, and PtALF from Portunus trituberculatus, have been reported to be expressed constitutively in hemocytes and up-regulated after bacteria challenges, which suggest that ALFs might play important roles in defense against bacterial pathogens [3-8]. The recombinant proteins of ALF genes by prokaryotic expression system exerted strong antibacterial effects on both Gram-positive and Gram-negative bacteria [4,5,8,9]. The recombinant ALF from P. monodon also showed antiviral activities against herpes simplex virus type 1 and human adenovirus respiratory strain in cultured mammalian cell lines [10] and white spot syndrome virus (WSSV) in cultured hematopoietic tissue (Hpt) cells from crayfish [11]. The expression of envelope protein VP28 of WSSV could be enhanced in the cultured Hpt cells from crayfish Pacifastacus leniusculus after ALF gene was interfered, and the mortality of LvALF1 knockdown shrimp increased significantly after $V$. penaeicida, Fussarium oxysporum or WSSV infections [3,12].

Different ALFs have a conserved cluster of positively charged residues within their disulfide loop between two conserved cysteine residues, which is usually called lipopolysaccharide (LPS)-binding domain [13-15]. The typical structure has been reported to be closely associated with the biological activities of ALFs and considered to be the vital functional domain [3,7,13,16-19]. The synthetic peptides corresponding to these LPS-binding domains, such as ALFSp, SsALF from Scylla paramamosain and Scylla serrata [17,20], and SALF55-76 from P. monodon [21] showed antibacterial activity against various bacteria. The synthetic LPS-binding domain from crayfish ALF could inhibit the replication of WSSV in cultured Hpt cells [11].

In our previous studies, the synthetic LBD peptides of two FcALF isoforms (FCALF2 and $A L F F c)$ were also found to inhibit bacterial growth and WSSV in vivo replication [22,23]. Further structure-activity analysis showed that their antibacterial activities were closely related with the disulfide bonds and basic amino acid residues in LBD peptides, while their anti-WSSV activities were associated with lysine residues [22,23]. In crustaceans, several ALF isoforms always coexist in one organism. In P. monodon, six ALF isoforms were identified by EST approach [7]. In P. trituberculatus, seven ALF isoforms were cloned and they displayed different biological activities [4-6,24,25]. Seven 
ALF isoforms (FcALF1-6 and ALFFc) were also identified in the Chinese shrimp Fenneropenaeus chinensis [26,27]. However, a comprehensive understanding on the diverse functions of these FcALFs is still very limited. In the present study, the functions of different FcALFs will be analyzed comparatively. These data will not only provide us a comprehensive understanding on the function of ALFs, but also give an instruction for the development of therapeutic drugs to shrimp disease control.

\section{Results}

\subsection{Tissue Distribution of Different FcALFs Transcripts}

In order to have a basic understanding on their potential function, tissue distribution of $F c A L F s$ was performed. Different $F C A L F S$ showed their various expression profiles. FCALF1 was prominently expressed in stomach, followed by Oka. Remarkably lower expression levels were detected in nerve cord, intestines, and gill. No expression was detected in muscle, hepatopancreas, hemocytes, heart, and eyestalk (Figure 1A). FcALF2 was mainly expressed in Oka, followed by nerve cord, gill, and eyestalk (Figure 1B). FCALF3 was also mainly expressed in Oka (Figure 1C). Both FCALF4 and FcALF5 were mainly detected in eyestalk and also showed high expression levels in Oka (Figure 1D,E), while FCALF5 was also highly expressed in hemocytes (Figure 1E). FCALF6 was detected in all tested tissues. The relatively high expression levels of FCALF6 were present in Oka, nerve cord, hemocytes and gill (Figure 1F). ALFFC was mainly detected in Oka and hemocytes (Figure 1G).

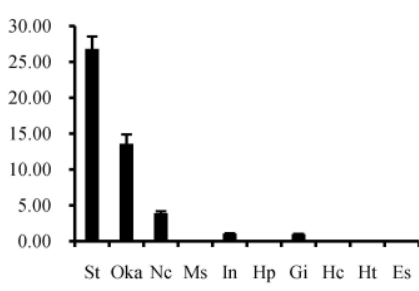

(A)

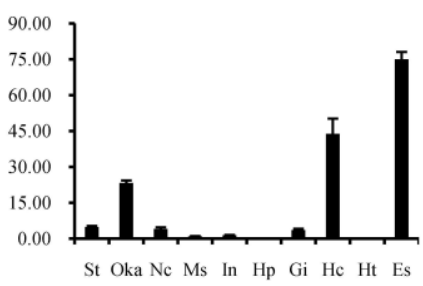

(E)

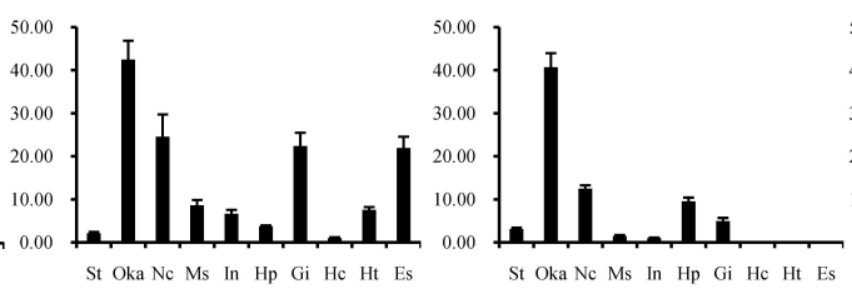

(B)

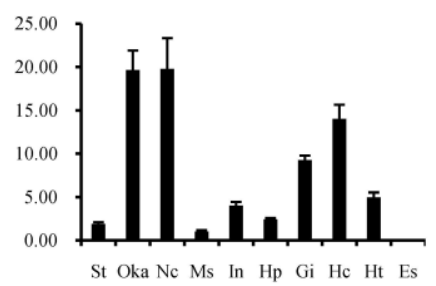

(F)

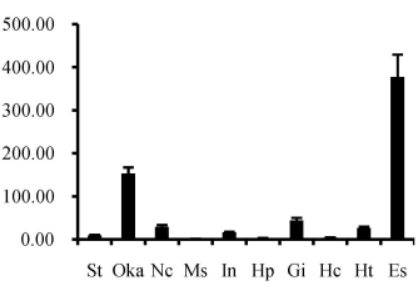

(D)

Figure 1. The tissue distribution of different FCALFs transcripts. FCALF1, FCLAF2, FCLAF3, FCLAF4, FCLAF5, FCLAF6 and ALFFC were shown in (A-G), respectively. St, Stomach; oka, lymphoid organ; Nc, nerve cord; Ms, muscle; In, intestine; Hp, hepatopancreas; Gi, gill; Hc, haemocytes; He, heart; Es, eyestalk. Vertical bars represented the mean \pm standard error $(\mathrm{SE})(n=10)$. 


\subsection{Antibacterial Activities of Synthetic LBD Peptides}

In order to know if FcALFs had antibacterial activities, inhibition zones and minimal inhibitory concentration (MIC) were examined using synthetic LBD peptides. Obvious inhibition zones with different sizes to M. luteus appeared around the filter paper that was loaded with LBD1, LBD2 and LBD3 peptide solutions, which correspond with that for positive controls of ampicillin (Amp+). For E. coli, LBD1, LBD4, LBD6 and LBD7-loaded paper also exhibited apparent inhibition zones. For B. simplex, similar inhibition zones were observed in LBD2, LBD5 and LBD7. Only LBD2 and LBD7-loaded paper exhibited inhibition zones in A. hydrophila plates. In addition, no inhibition zones appeared for negative control pGFP and blank control phosphate-buffered saline (PBS) either (Figure 2).
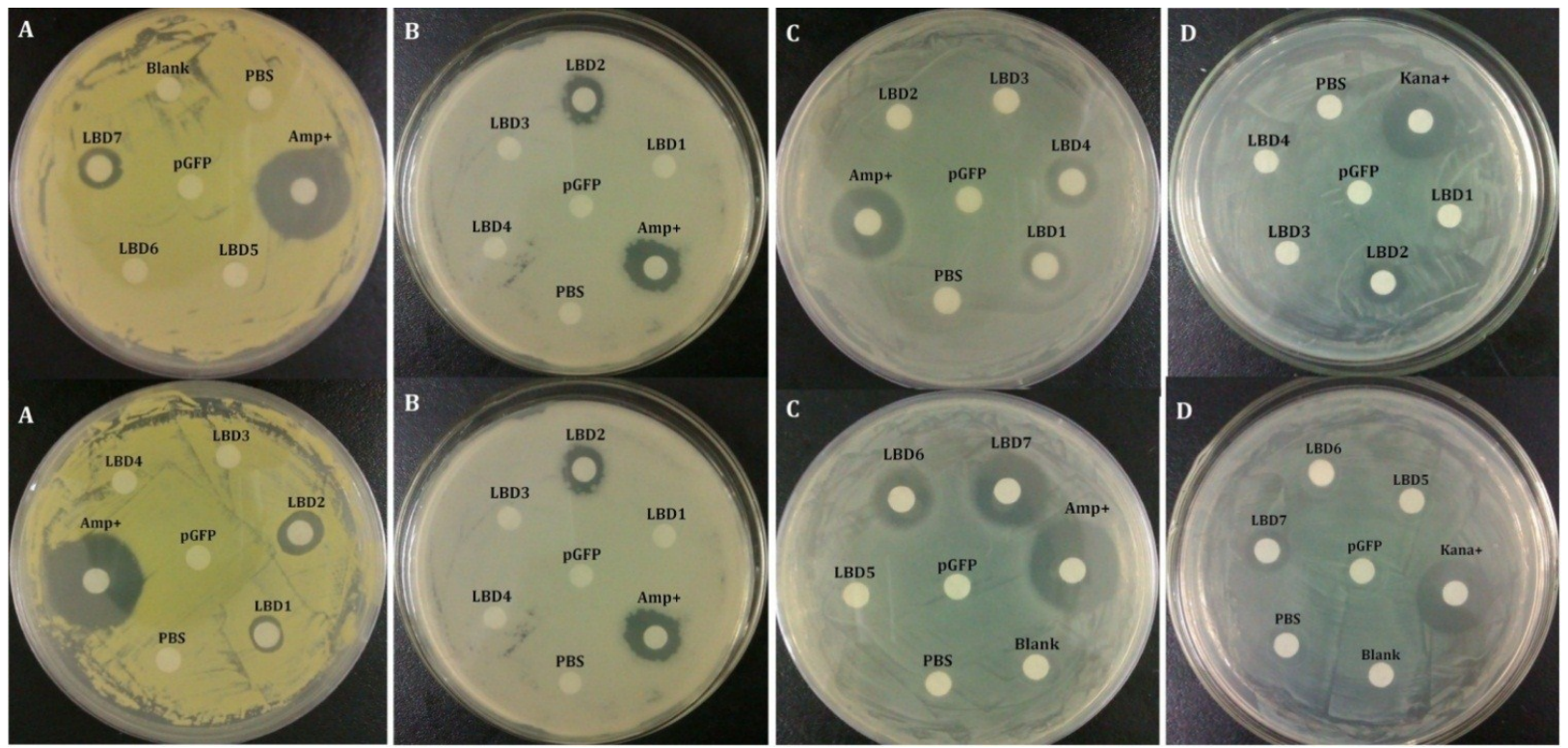

Figure 2. Inhibition zones of synthetic peptides corresponding to LBDs of different FcALFs. "Blank" represented blank group with nothing added. "PBS" represented control group with only PBS. "pGFP" represented negative control with synthetic pGFP peptide. "Amp+" and "Kana+" were positive controls with ampicillin and kanamycin sulfate separately. The bacterium strains M. luteus (A); B. simplex (B); E. coli (C); and A. hydrophila (D) were used for antibacterial analyses.

The antimicrobial activities of the synthetic LBD peptides were tested by measuring the MICs quantitatively. The inhibition of bacterial growth was considered when $\mathrm{OD}_{600}$ was significantly lower than that of PBS control. The data demonstrated that LBD1 effectively inhibited the growth of $M$. luteus and E. coli with the same MIC ranges of 16-32 $\mu \mathrm{M}$. LBD2 could inhibit the growth of $M$. luteus and B. simplex, more effectively than E. coli and A. hydrophila. The MIC ranges of LBD2 peptide for M. luteus and B. simplex were 2-4 $\mu \mathrm{M}$ and 1-2 $\mu \mathrm{M}$, respectively, while for A. hydrophila, the MIC was 32-64 $\mu \mathrm{M}$. No antibacterial activity against four tested bacteria was observed for LBD3 peptide even at $64 \mu \mathrm{M}$. LBD4 and LBD6 were specifically against $E$. coli with the MIC ranges of 8-16 $\mu \mathrm{M}$ and 16-32 $\mu \mathrm{M}$, respectively. LBD5 was specifically against $B$. simplex with the MIC range of 16-32 $\mu \mathrm{M}$. Among the seven LBD peptides, LBD7 showed the most effective antibacterial activity 
with the broadest spectrum. It could inhibit the growth of both Gram-negative bacteria $E$. coli and A. hydrophila and Gram-positive bacteria $M$. luteus and B. simplex, especially against $M$. luteus and A. hydrophila with MIC ranges of $1-2 \mu \mathrm{M}$ and $2-4 \mu \mathrm{M}$, respectively. The MICs of different peptides were shown in Table 1.

Table 1. Minimal inhibitory concentration of synthetic LPS-binding domain peptides on different bacterium strains.

\begin{tabular}{|c|c|c|c|c|c|c|c|}
\hline \multirow{2}{*}{ Bacteria } & \multicolumn{7}{|c|}{ MIC Range $(\boldsymbol{\mu M})$} \\
\hline & LBD1 & LBD2 & LBD3 & LBD4 & LBD5 & LBD6 & LBD7 \\
\hline M. luteus & $16-32$ & $2-4$ & $>64$ & $>64$ & $>64$ & $>64$ & $1-2$ \\
\hline B. simplex & $>64$ & $1-2$ & $>64$ & $>64$ & $16-32$ & $>64$ & $32-64$ \\
\hline E. coli & $16-32$ & $>64$ & $>64$ & $8-16$ & $>64$ & $16-32$ & $32-64$ \\
\hline A. hydrophila & $>64$ & $32-64$ & $>64$ & $>64$ & $>64$ & $>64$ & $2-4$ \\
\hline
\end{tabular}

\subsection{Effects of Synthetic LBD Peptides of FcALFs on WSSV Infection}

In order to know if FcALFs could inhibit WSSV replication, WSSV pre-incubated with synthetic LBD peptides was injected into E. carinicauda and the in vivo WSSV copy number was tested. As shown in Figure 3, different LBD peptides showed distinct impacts on the WSSV infection. The background value of WSSV copy number in the blank group was $1.31 \times 10^{3}$ copies/ng DNA. In WSSV group, the WSSV copy number reached $1.21 \times 10^{5}$ copies/ng DNA, which had no significant difference with that in pGFP group $\left(1.08 \times 10^{5}\right.$ copies/ng DNA). After incubation with LBD1, LBD2, LBD5 and LBD7, the copy numbers of WSSV in shrimp were obviously restricted. The WSSV copy number in LBD1, LBD2, LBD5 and LBD7 group were $4.36 \times 10^{3}$ copies/ng DNA, $1.40 \times 10^{3}$ copies/ng DNA, $2.07 \times 10^{3}$ copies/ng DNA and $5.07 \times 10^{3}$ copies/ng DNA, respectively, which were significantly lower than those in WSSV group and pGFP group. The WSSV copy number in LBD3, LBD4 and LBD6 group were $1.19 \times 10^{5}$ copies/ng DNA, $7.24 \times 10^{4}$ copies/ng DNA and $1.55 \times 10^{5}$ copies/ng DNA, which had no significant differences with those in WSSV group and pGFP group.

\subsection{Relationship between Antiviral Activity and the Sequence Feature of LBD Peptides}

In order to find out if LBD peptides with anti-WSSV activities had similar characteristics, multiple alignments among them were performed. Data showed that sequence similarity among seven LBD peptides was very low and only three identical amino acid residues, two cysteine residues and one proline residue, were shared by them (Figure 4A). Taken the acid-base properties of the amino acid residues into consideration, higher similarity existed among seven LBD peptides, where a consensus sequence $(2 . .2 . .1 .1 . .1 .1 \ldots . .1 .2$, where 2 presented hydrophobic amino acid residues and 1 presented neutral amino acid residues) with eight identical sites were found (Figure 4B). One lysine site was identical among four LBD peptides with anti-WSSV activity, including LBD-1, LBD-2, LBD-5 and LBD-7, while not found at this site in other peptides (showed by an arrow in Figure 2A and the left arrow in Figure 4B). Besides, another identical basic amino acid site (showed by the right arrow in Figure 4B) only existed among four LBD peptides with anti-WSSV activity. 


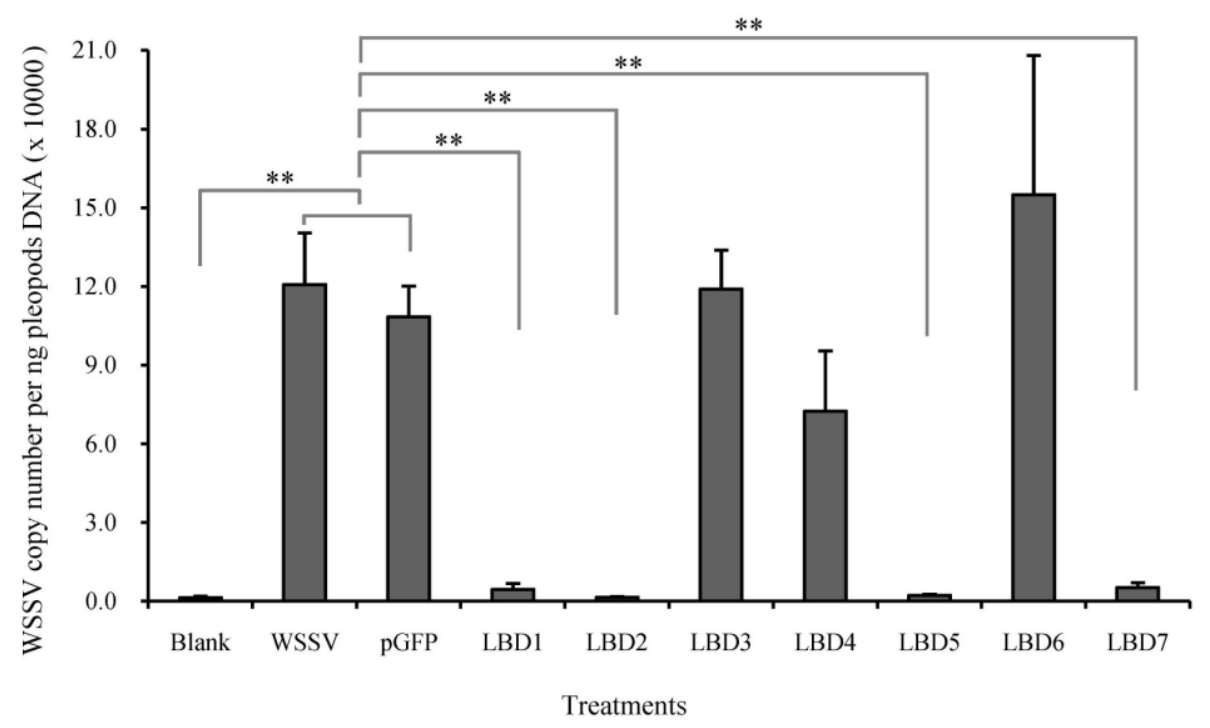

Figure 3. Comparison of viral propagation in E. carinicauda after infection with pre-incubated WSSV in synthetic peptides corresponding to LBDs of different FcALFs. "Blank" was group without WSSV or peptide injection and "WSSV" was group injected with WSSV. $n=15$. Stars $(* *)$ indicated significant differences at $P<0.01$ between different treatments.

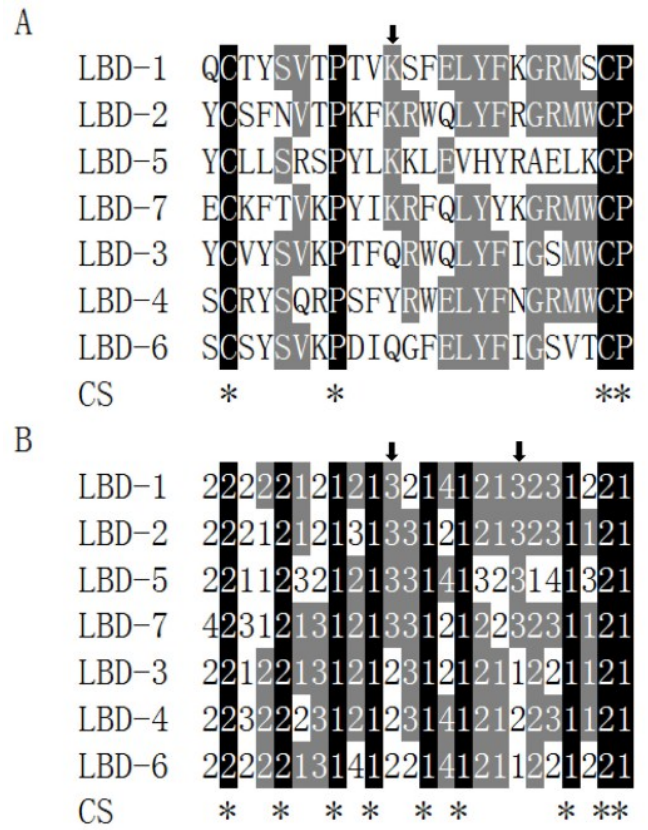

Figure 4. Sequence analyses of LBD peptides of different FcALFs. (A) Showed multiple alignments of LBD sequences from seven ALFs in F. chinensis; (B) Showed multiple alignments of these LBD sequences base on the acid-base properties of amino acid residues. "1", "2", “3" and "4" represented hydrophobic, neutral, basic and acidic amino acid residues, respectively. CS represented consensus sequence. Identical sites were marked by black background and shown with stars below. Sites with a similarity more than $50 \%$ were highlighted with gray background. Arrows showed identical sites that only existed in anti-WSSV peptides. 


\subsection{Effects of Modified LBD Peptides on WSSV Infection}

From the sequence analysis results, we found that an identical lysine residue was important for the antiviral activity of LBD peptides. In order to verify the hypothesis, three new sequence-modified peptides were synthesized and named as SLBD2, LBD2-K and LBD4+K, respectively. The anti-WSSV effect of these peptides was detected and the result was shown in Figure 5. The WSSV copy number in sLBD2 treated group was $4.04 \times 10^{3}$ copies/ng DNA, which was significantly lower than those in WSSV group and pGFP group, while no difference was found in SLBD2 group and LBD2 group. The WSSV copy number in LBD2-K treated group was $5.14 \times 10^{4}$ copies/ng DNA, which was significantly higher than that in LBD2 group. The WSSV copy number in LBD4+K treated group was $3.40 \times 10^{3}$ copies/ng DNA, which was significantly lower than those in WSSV group, pGFP group and LBD4 group.

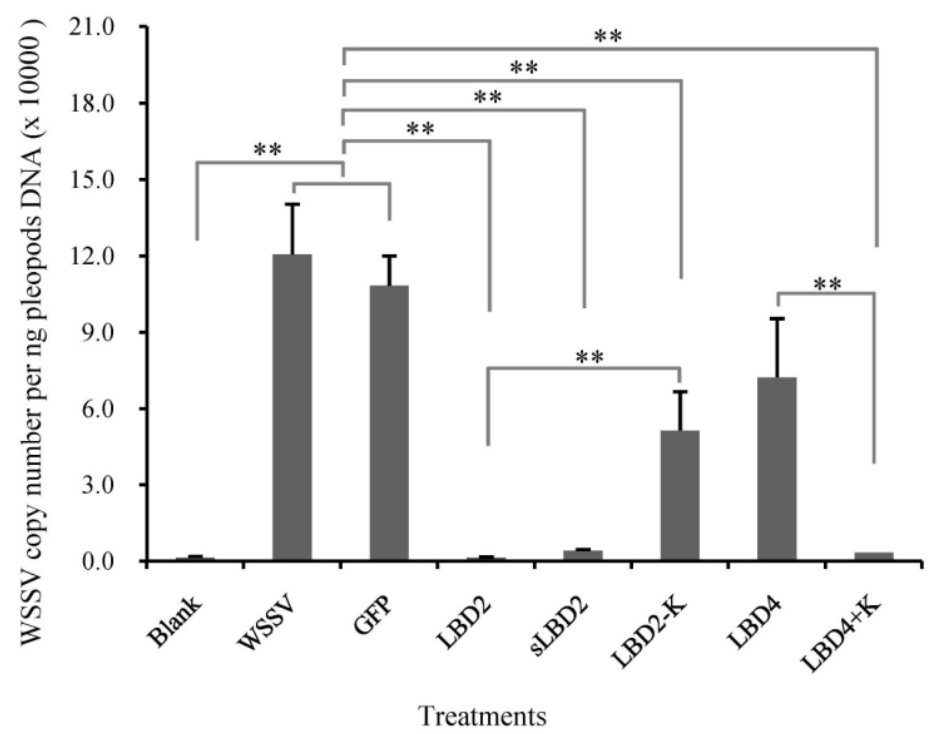

Figure 5. Comparison of viral propagation in E. carinicauda after infection with pre-incubated WSSV in modified peptides. Viral propagation was shown by measuring the expression level of VP28 in E. carinicauda at $24 \mathrm{~h}$ after infection of pre-incubated WSSV with $50 \mu \mathrm{M}$ different peptides. "Blank" was group without WSSV or peptide injection and "WSSV" was group injected with WSSV. $n=15$. Stars $(* *)$ indicated significant differences at $P<0.01$ between different treatments.

\section{Discussions}

In the present study, we characterized seven types of ALF from the Chinese shrimp $F$. chinensis. Two of them, $F C A L F 2$ and $A L F F c$, have been reported in our previous studies [22,23]. Here we characterized and compared the distribution and activities of all ALF isoforms in F. chinensis in detail.

Tissue distribution of the gene is always related with its biological function [28]. Seven ALFs exhibited diverse tissue distribution patterns and various antimicrobial properties. It was noticeable that all ALFs displayed high expression level in the lymphoid organ (Oka). FCALF5, FCALF6 and $A L F F C$ also showed high expression level in hemocytes, while FCALF2, FCALF4 and FCALF5 were 
also detected at a high expression level in eyestalk and FCALF1 was highly expressed in stomach. Oka is deemed to play an important role in the innate immune system [29], and is the most specific and effective organ for clearance of bacteria [30] and virus [31] in penaeid shrimp. Hemocytes play important roles in the host immune response including recognition, phagocytosis, melanization, cytotoxicity and cell-cell communication [32]. Stomach was found as a target tissue for WSSV infection [33]. It is very curious that some ALF isoforms were highly expressed in eyestalk, which was always regarded as a neuroendocrine organ [34]. However, recent studies revealed that the eyestalk might contribute to crustacean immunity [24] and neuropeptides release from eyestalk to hemolymph was important for WSSV replication by elevating glycolytic activity and glucose mobilization [35]. A multiple and high expression patterns of FCALFs in these organs indicated that they might be participated in shrimp immune defense against distinct pathogens.

Previously, we found that FcALF2 exhibited strong inhibition activities against WSSV and Gram-positive bacteria, M. luteus and M. lysodeikticus, but poorly active against Gram-negative bacteria, E. coli and $V$. anguillarum [23]. However, ALFFc showed a quite different antimicrobial spectrum, which strongly inhibited WSSV, Gram-positive and Gram-negative bacteria [22]. In order to know the antimicrobial activities of other FcALFs, we synthesized the peptides corresponding to LPS-binding domains of these FcALFs. All synthetic peptides displayed antibacterial activities against one or more kinds of bacteria except LBD3, which showed no effect to the four tested bacteria species. Due to the limited number of tested bacteria species, it was not sure whether LBD3 inhibited the growth of other bacteria. It was also reported that different ALFs in other species showed distinct antimicrobial properties. In the swimming crab P. trituberculatus, six recombinant PtALF proteins could inhibit the growth of distinct Gram-positive, Gram-negative bacteria or Fungi $[5,6,24,25]$. The multiple antimicrobial properties of ALFs suggested that they might defense various pathogens synergistically in an organism.

ALF proteins were also reported to have antiviral activity. The recombinant ALF protein of P. monodon was found inhibiting herpes simplex virus type 1, human adenovirus respiratory strain and WSSV replication [10,11]. In our study, the LBD peptides from four of the FcALFs, including FcALF1, FcALF2, FcALF5 and ALFFc, could inhibit WSSV replication in experimental animals. Loss of antiviral function could be directly caused by replacing lysine residues with other amino acid residues in ALFFc-LBD peptide [22], which suggested that lysine residues in LBD peptides were important for their antiviral activities. However, increasing the number of lysine residues in LBD peptide did not show a better anti-WSSV performance [23], which might be due to the high peptides concentration $(50 \mu \mathrm{M})$ concealed the difference among LBD peptides. Further sequence analysis discovered a conserved lysine residue site that only existed in LBD peptides with anti-WSSV activities. Interestingly, replacing this lysine residue with other amino acid residue in LBD peptide with anti-WSSV activity could lead to a considerable reduction of their anti-WSSV function, while replacing other residue with a lysine residue at this site in LBD peptide without anti-WSSV activity could endow them with anti-WSSV ability. The data proved that the certain lysine residue played a key role in inhibiting WSSV replication, which provided insight into understanding how ALFs exerted their anti-WSSV functions.

In conclusion, the present study compared the tissue distribution and antimicrobial activities of seven ALFs from the Chinese shrimp F. chinensis. Different ALFs possessed distinct antimicrobial 
activities. Sequence analysis and further function confirmation found that a certain lysine residue in LBD peptides is vital for their anti-WSSV activities. These results not only showed us a comprehensive understanding on the function of ALFs from $F$. chinensis, but also provided clues to development of ALFs as potential therapeutic drugs.

\section{Materials and Methods}

\subsection{Animal and Tissue Collection}

Healthy shrimp, F. chinensis, with a body weight of $38.97 \pm 4.34 \mathrm{~g}$, were acclimatized in the aerated seawater at $25{ }^{\circ} \mathrm{C}$ for three days before tissues collection. Hemolymph from ten individuals was collected from the ventral sinus located at the first abdominal segment with an equal volume of anticoagulant-modified Alsever solution ( $27 \mathrm{mM}$ sodium citrate, $336 \mathrm{mM} \mathrm{NaCl}, 115 \mathrm{mM}$ glucose, $9 \mathrm{mM}$ EDTA, pH 7) [36]. Hemocytes from ten individuals were isolated by centrifugation at $800 \mathrm{~g}$, $4{ }^{\circ} \mathrm{C}$ for $10 \mathrm{~min}$ and then preserved in liquid nitrogen. Other tissues including stomach, lymphoid organ (Oka), nerve cord, muscle, intestines, hepatopancreas, gill, heart, and eyestalk were dissected and preserved in liquid nitrogen respectively for RNA extraction and gene expression analysis.

Exopalaemon carinicauda is being regarded as a good crustacean model animal for WSSV infection experiments [37]. In this study, healthy E. carinicauda with a body length of $5.43 \pm 0.30 \mathrm{~cm}$ and a body weight of $2.62 \pm 0.42 \mathrm{~g}$ were used as experimental animals for WSSV infection assay. Animals were acclimatized in the aerated seawater at $25^{\circ} \mathrm{C}$ for 1 day before experiments.

\subsection{Total RNA Extraction, cDNA Synthesis and qPCR Analysis}

The total RNA from each sample was extracted using Unizol reagent (BiostarGenechip, Shanghai, China). The first-strand cDNA was synthesized with M-MLV reverse transcriptase (Promega, Fitchburg, WI, USA) and oligodT primer.

The expression level of $F c A L F s$ in different tissues were detected by quantitative real-time RT-PCR with corresponding primers (Table 2) using a Master Cyclereprealplex (Eppendorf, Hamburg, Germany). The RT-PCR program was set as follows: $95{ }^{\circ} \mathrm{C}$ for $2 \mathrm{~min}$, followed by 40 cycles of $94{ }^{\circ} \mathrm{C}$ for $20 \mathrm{~s}$, annealing temperature for $20 \mathrm{~s}$ and $72{ }^{\circ} \mathrm{C}$ for $20 \mathrm{~s}$. The expression level of $18 \mathrm{~S}$ rRNA was used as an internal reference standard. The dissociation curve analysis of amplification products was performed at the end of each PCR reaction to confirm the specificity of the amplification. Then the data was analyzed by $2^{-\Delta \Delta C_{\mathrm{T}}}$ method [38] to calculate the relative gene expression levels.

\subsection{Multiple Alignments of Synthetic LBD Peptides}

Multiple alignments of synthetic LBD1, LBD2, LBD3, LBD4, LBD5, LBD6 and LBD7 peptides were carried out based on the sequences and the acid-base properties of the amino acid residues, respectively. 
Table 2. Primers used for gene cloning and qPCR analysis.

\begin{tabular}{|c|c|c|c|}
\hline Gene & Primer Name & Sequence $\left(5^{\prime}-3^{\prime}\right)$ & Annealing Temperature $\left({ }^{\circ} \mathrm{C}\right)$ \\
\hline \multirow{2}{*}{ FcALF1 } & FcALF1-qF & ATGTCCTGCCCGTCCCTTAG & \multirow{2}{*}{59} \\
\hline & FcALF1-qR & CCTCCGTTATCACGCCCTGT & \\
\hline \multirow{2}{*}{ FcALF2 } & FcALF2-qF & TGCGAGTGTCAGTCTTTAGC & \multirow{2}{*}{61} \\
\hline & FcALF2-qR & CAATCCTGTGAGTTTGTCCG & \\
\hline \multirow{2}{*}{ FcALF3 } & FcALF3-qF & CAGGATTGTGGGAGACGGGA & \multirow{2}{*}{61} \\
\hline & FcALF3-qR & CTGCTGCGTGTTTCGGCTAC & \\
\hline \multirow{2}{*}{ FcALF4 } & FcALF4-qF & ACGATGCGAGTCTTGGTCAG & \multirow{2}{*}{59} \\
\hline & FcALF4-qR & CTCATCCGAGTGCCACAACC & \\
\hline \multirow{2}{*}{ FcALF5 } & FcALF5-qF & GCTTGTTGAGTCGCAGTCCT & \multirow{2}{*}{59} \\
\hline & FcALF5-qR & GAGCCTGTCTTATGAAATCCTT & \\
\hline \multirow{2}{*}{ FcALF6 } & FcALF6-qF & AGACTTATGGAGGAACGGAGAC & \multirow{2}{*}{60} \\
\hline & FcALF6-qR & ATTTGCTGCGGGTGTTGGAC & \\
\hline \multirow{2}{*}{ ALFFc } & ALFFc-qF & AAGCCTGGTGCTGGTGGTGT & \multirow{2}{*}{59} \\
\hline & ALFFc-qR & GAGTTCGGTTTTCTCGTTCCT & \\
\hline \multirow{2}{*}{$18 \mathrm{~S}$ rRNA } & $18 \mathrm{~S}-\mathrm{qF}$ & TATACGCTAGTGGAGCTGGAA & \multirow{2}{*}{56} \\
\hline & $18 \mathrm{~S}-\mathrm{qR}$ & GGGGAGGTAGTGACGAAAAAT & \\
\hline
\end{tabular}

\subsection{Synthesis of Different LBD Peptides of FcALF Isoforms}

Seven peptides corresponding to the LPS-binding domain (LBD) of different FcALF isoforms (Accession numbers of these genes were listed in Table 3) with one flanking amino acid residue in each $\mathrm{N}$ and $\mathrm{C}$ terminal were synthesized. They were named as LBD1-7, respectively. A disulfide bond was formed between two cysteine residues in each peptide. According to our previous studies [22,23], the peptides corresponding to LBDs of FcALF2 and ALFFc were used as positive control. Another peptide with 24 amino acid residues based on the amino acid sequence of Green Fluorescent Protein (pGFP) was also synthesized as a negative control. Based on the results of our functional experiments, another three peptides, LBD2-K, sLBD2 and LBD4+K, were also synthesized. The sequence of LBD2-K was the same as LBD2 except for the replacement of a lysine residue by a glutamine residue. The peptide sLBD2 was a part (ten amino acid residues) of LBD. The sequence of LBD4+K was the same as LBD4 except for the replacement of a tyrosine residue by a lysine residue. All peptides were acetylated in the $\mathrm{N}$ terminal and amidated in the $\mathrm{C}$ terminal. These peptides were all synthesized in Ziyu Biotechnology Co. Ltd. (Shanghai, China). The detailed sequence information of above synthesized peptides was shown in Table 3.

\subsection{Inhibition Zone Test}

Gram-negative bacteria, including Escherichia coli and Aeromonas hydrophila, and Gram-positive bacteria, including Micrococcus luteus and Bacillus simplex were used for antibacterial analyses. The strains were incubated in fresh LB medium overnight at $37{ }^{\circ} \mathrm{C}$ for E. coli, M. luteus and $28^{\circ} \mathrm{C}$ for A. hydrophila, B. simplex. 
Table 3. Sequence information of synthetic peptides.

\begin{tabular}{|c|c|c|c|}
\hline Gene Name & Accession Number & Peptide Name & Sequence \\
\hline FcALF1 & JX853774 & LBD1 & Ac-Q(CTYSVTPTVKSFELYFKGRMSC)P-NH ${ }_{2}$ \\
\hline FcALF2 & JX853775 & LBD2 & Ac-Y(CSFNVTPKFKRWQLYFRGRMWC)P-NH \\
\hline FcALF3 & JX853776 & LBD3 & Ac-Y(CVYSVKPTFQRWQLYFIGSMWC)P-NH \\
\hline FcALF4 & JX853777 & LBD4 & Ac-S(CRYSQRPSFYRWELYFNGRMWC)P-NH \\
\hline FcALF5 & JX853778 & LBD5 & Ac-Y(CLLSRSPYLKKLEVHYRAELKC)P-NH \\
\hline FcALF6 & JX853779 & LBD6 & Ac-S(CSYSVKPDIQGFELYFIGSVTC)P-NH ${ }_{2}$ \\
\hline $\mathrm{ALFFc}$ & AY859500 & LBD7 & Ac-E(CKFTVKPYIKRFQLYYKGRMWC)P-NH ${ }_{2}$ \\
\hline l & l & LBD2-K & Ac-Y(CSFNVTPKFQRWQLYFRGRMWC)P-NH \\
\hline / & l & sLBD2 & Ac-FKRWQLYFRG-NH ${ }_{2}$ \\
\hline / & 1 & $\mathrm{LBD} 4+\mathrm{K}$ & Ac-S(CRYSQRPSFKRWELYFNGRMWC)P-NH ${ }_{2}$ \\
\hline GFP & AAN41637 & pGFP & Ac-TTGKLPVPWPTLVTTFSYGVQCFS-NH ${ }_{2}$ \\
\hline
\end{tabular}

Note: "Ac-"represents acetylation of the $N$-terminal amino acid residue; "-NH2" represents amidation of the $C$-terminal amino acid residue; parentheses at two sides of Cysteine amino acids shows a disulfide bond.

The antibacterial activities of LBD peptides were examined by inhibition zone test. The overnight cultures of bacteria were diluted 100 times and cultured in LB medium at $37{ }^{\circ} \mathrm{C}$ (E. coli and M. luteus) or $28{ }^{\circ} \mathrm{C}$ (A. hydrophila and B. simplex) for $6 \mathrm{~h}$. These cultures were then diluted 100 times with LB medium separately, and $200 \mu \mathrm{L}$ solutions from each diluted cultures was taken out and spread on the solid LB medium plate uniformly. Sterile filter paper with a diameter of $6 \mathrm{~mm}$ was put on the surface of solid LB medium gently. Twenty microliters of $64 \mu \mathrm{M}$ peptide solution dissolved in PBS (pH 7.4) was added to the center of filter paper with a diameter of $6 \mathrm{~mm}$. Twenty microliters PBS and $20 \mu \mathrm{L}$ of $64 \mu \mathrm{M}$ pGFP peptide were used as negative controls. Fifty microliters ampicillin solutions was used as the positive control for E. coli, M. luteus and B. simplex culture, and $50 \mu \mathrm{M}$ kanamycin sulfates was used as the positive control for $A$. hydrophila culture. The plates were cultured at $37{ }^{\circ} \mathrm{C}($ E. coli and M. luteus) or $28^{\circ} \mathrm{C}$ (A. hydrophila and B. simplex) for $24 \mathrm{~h}$.

\subsection{Minimal Inhibitory Concentration (MIC) Assay}

MIC assay was performed according to the method described by Wiegand et al. [39] with slight modification. The bacterial strains were culture in LB medium at $37{ }^{\circ} \mathrm{C}$ (E. coli and M. luteus) or $28{ }^{\circ} \mathrm{C}$ (A. hydrophila and B. simplex) till the $\mathrm{OD}_{600}$ reached certain values corresponding to $\sim 1 \times 10^{8}$ colony forming units $(\mathrm{CFU}) / \mathrm{mL}$. Then, $2 \mu \mathrm{L}$ of the bacterial cultures, $20 \mu \mathrm{L}$ of $1 / 2$-fold serially diluted peptides $(640 \mu \mathrm{M}-10 \mu \mathrm{M})$ in PBS (pH 7.4), and $178 \mu \mathrm{L}$ of fresh LB medium were added into each well of sterile 48 -well plates. The final concentration of the bacterial cultures was diluted to $\sim 1 \times 10^{6} \mathrm{CFU} / \mathrm{mL}$. The peptides solutions were diluted at $1 / 2$-fold serial concentrations from $64 \mu \mathrm{M}$ to $1 \mu \mathrm{M}$ in a final volume of $200 \mu \mathrm{L}$. The 48 -well plates were incubated at $37{ }^{\circ} \mathrm{C}($ E. coli and M. luteus) or $28^{\circ} \mathrm{C}$ (A. hydrophila and B. simplex) for $3 \mathrm{~h}$. Then, $300 \mu \mathrm{L}$ of fresh LB medium was added and the plates were incubated at the corresponding temperatures for another $18 \mathrm{~h}$. The optical density (OD) was measured at $600 \mathrm{~nm}\left(\mathrm{OD}_{600}\right)$ using a micro-plate reader (TECAN infinite M200 PRO, Austria). PBS and pGFP at the same concentration with LBD peptides were used as negative controls. Antibiotics (50 $\mu \mathrm{M}$ ampicillin for E. coli, M. luteus, and B. simplex, $50 \mu \mathrm{M}$ kanamycin for A. hydrophila) were used as positive controls. The assay was performed in triplicates. 


\subsection{Antiviral Activity Detection}

In order to learn the effect of synthetic LBD peptides on WSSV infection, WSSV pre-incubated with different peptides was injected into E. carinicauda and the WSSV copy number in the pleopods of E. carinicauda was detected. E. carinicauda was classified into 13 groups and each group contained 18 to 20 individuals. The 13 groups were named as Blank, WSSV, pGFP, LBD1, LBD2, LBD3, LBD4, LBD5, LBD6, LBD7, sLBD2, LBD2-K and LBD4+K, respectively. Synthetic peptides were diluted into $50 \mu \mathrm{M}$ with PBS ( $\mathrm{pH}$ 7.4). WSSV was incubated with each peptide solution for $2 \mathrm{~h}$ at room temperature with a final concentration $10^{3}$ copies $/ \mu \mathrm{L}$. For Blank group, shrimp did not suffer any injection. For WSSV group, each shrimp was injected with $10 \mu \mathrm{L}$ WSSV incubated with PBS (pH 7.4) for $2 \mathrm{~h}$ at room temperature. For the other groups (LBD1-LBD7, sLBD2, LBD2-K and LBD4+K), each shrimp was injected with $10 \mu \mathrm{L}$ of WSSV solution after incubation with corresponding synthetic peptides, respectively. At $24 \mathrm{~h}$ post injection (hpi), the pleopods of 15 shrimps in each group were collected and the pleopods from three individuals were put together as one sample. Total DNA of each sample was extracted using the Genomic DNA Kit (Tiangen, China). The viral copy number was measured by detecting the level of VP28 using real-time PCR as described previously [37].

\subsection{Statistical Analysis}

All data were given in the form of mean \pm SE and analyzed with one-way analysis of variance (ANOVA) and Ducan's Multiple Comparisons. Differences between treatments and controls were considered significant at $p<0.05$.

\section{Acknowledgments}

This work was financially supported by the National High Technology Research and Development Program of China (863 Program, No. 2014AA093501), Major State Basic Research Development Program (2012CB114403), National Natural Science Foundation Program (31272683), China Agriculture Research System-47 (CARS-47), and Special Fund for Agro-scientific Research in the Public Interest (201103034).

\section{Author Contributions}

Conceived and designed the experiments: S.L., F.L., J.X. Performed the experiments: S.L., S.G. Analyzed and discussed the data: S.L., S.G., F.L. Wrote the paper: S.L., S.G., F.L.

\section{Conflicts of Interest}

The authors declare no conflict of interest.

\section{References}

1. Morita, T.; Ohtsubo, S.; Nakamura, T.; Tanaka, S.; Iwanaga, S.; Ohashi, K.; Niwa, M. Isolation and biological-activities of limulus anticoagulant (anti-LPS factor) which interacts with lipopolysaccharide (LPS). J. Biochem. 1985, 97, 1611-1620. 
2. Tanaka, S.; Nakamura, T.; Morita, T.; Iwanaga, S. Limulus Anti-LPS Factor: An anticoagulant which inhibits the endotoxin-mediated activation of limulus coagulation system. Biochem. Biophys. Res. Commun. 1982, 105, 717-723.

3. De la Vega, E.; O’Leary, N.A.; Shockey, J.E.; Robalino, J.; Payne, C.; Browdy, C.L.; Warr, G.W.; Gross, P.S. Anti-lipopolysaccharide factor in Litopenaeus vannamei (LvALF): A broad spectrum antimicrobial peptide essential for shrimp immunity against bacterial and fungal infection. Mol. Immunol. 2008, 45, 1916-1925.

4. Liu, Y.; Cui, Z.X.; Luan, W.S.; Song, C.W.; Nie, Q.; Wang, S.Y.; Li, Q.Q. Three isoforms of anti-lipopolysaccharide factor identified from eyestalk cDNA library of swimming crab Portunus trituberculatus. Fish Shellfish Immunol. 2011, 30, 583-591.

5. Liu, Y.; Cui, Z.X.; Li, X.H.; Song, C.W.; Li, Q.Q.; Wang, S.Y. Molecular cloning, expression pattern and antimicrobial activity of a new isoform of anti-lipopolysaccharide factor from the swimming crab Portunus trituberculatus. Fish Shellfish Immunol. 2012, 33, 85-91.

6. Liu, Y.; Cui, Z.X.; Li, X.H.; Song, C.W.; Shi, G.H. A newly identified anti-lipopolysaccharide factor from the swimming crab Portunus trituberculatus with broad spectrum antimicrobial activity. Fish Shellfish Immunol. 2013, 34, 463-470.

7. Supungul, P.; Klinbunga, S.; Pichyangkura, R.; Hirono, I.; Aoki, T.; Tassanakajon, A. Antimicrobial peptides discovered in the black tiger shrimp Penaeus monodon using the EST approach. Dis. Aquat. Organ. 2004, 61, 123-135.

8. Zhang, Y.; Wang, L.; Wang, L.; Yang, J.; Gai, Y.; Qiu, L.; Song, L. The second anti-lipopolysaccharide factor (EsALF-2) with antimicrobial activity from Eriocheir sinensis. Dev. Comp. Immunol. 2010, 34, 945-952.

9. Somboonwiwat, K.; Marcos, M.; Tassanakajon, A.; Klinbunga, S.; Aumelas, A.; Romestand, B.; Gueguen, Y.; Boze, H.; Moulin, G.; Bachere, E. Recombinant expression and anti-microbial activity of anti-lipopolysaccharide factor (ALF) from the black tiger shrimp Penaeus monodon. Dev. Comp. Immunol. 2005, 29, 841-851.

10. Carriel-Gomes, M.C.; Kratz, J.M.; Barracco, M.A.; Bachere, E.; Barardi, C.R.M.; Simoes, C.M.O. In vitro antiviral activity of antimicrobial peptides against herpes simplex virus 1 , adenovirus, and rotavirus. Mem. Inst. Oswaldo Cruz 2007, 102, 469-472.

11. Tharntada, S.; Ponprateep, S.; Somboonwiwat, K.; Liu, H.; Söderhäll, I.; Söderhäll, K.; Tassanakajon, A. Role of anti-lipopolysaccharide factor from the black tiger shrimp, Penaeus monodon, in protection from white spot syndrome virus infection. J. Gen. Virol. 2009, 90, 1491-1498.

12. Liu, H.P.; Jiravanichpaisal, P.; Soderhall, I.; Cerenius, L.; Soderhall, K. Antilipopolysaccharide factor interferes with white spot syndrome virus replication in vitro and in vivo in the crayfish Pacifastacus leniusculus. J. Virol. 2006, 80, 10365-10371.

13. Hoess, A.; Watson, S.; Siber, G.R.; Liddington, R. Crystal-structure of an endotoxin-neutralizing protein from the horseshoe-crab, limulus anti-LPS factor, at 1.5 angstrom resolution. EMBO J. 1993, 12, 3351-3356.

14. Somboonwiwat, K.; Bachere, E.; Rimphanitchayakit, V.; Tassanakajon, A. Localization of anti-lipopolysaccharide factor (ALFPm3) in tissues of the black tiger shrimp, Penaeus monodon, and characterization of its binding properties. Dev. Comp. Immunol. 2008, 32, 1170-1176. 
15. Yang, Y.; Boze, H.; Chemardin, P.; Padilla, A.; Moulin, G.; Tassanakajon, A.; Pugnière, M.; Roquet, F.; Destoumieux-Garzón, D.; Gueguen, Y. NMR structure of rALF-Pm3, an anti-lipopolysaccharide factor from shrimp: Model of the possible lipid A-binding site. Biopolymers 2009, 91, 207-220.

16. Hancock, R.E.W.; Piers, K.; Brown, M.; Falla, T.; Gough, M.; Wu, M.H.; Fidai, S. Cationic peptides: A class of antibiotics able to access the self-promoted uptake pathway across the Pseudomonas aeruginosa outer membrane. In Molecular Biology of Pseudomonads; Nakazawa, T., Furukawa, K., Haas, D., Silver, S., Eds.; ASM Press: Washington, DC, USA, 1996; pp. 441-450.

17. Imjongjirak, C.; Amparyup, P.; Tassanakajon, A.; Sittipraneed, S. Antilipopolysaccharide factor (ALF) of mud crab Scylla paramamosain: Molecular cloning, genomic organization and the antimicrobial activity of its synthetic LPS binding domain. Mol. Immunol. 2007, 44, 3195-3203.

18. Nagoshi, H.; Inagawa, H.; Morii, K.; Harada, H.; Kohchi, C.; Nishizawa, T.; Taniguchi, Y.; Uenobe, M.; Honda, T.; Kondoh, M.; et al. Cloning and characterization of a LPS-regulatory gene having an LPS binding domain in kuruma prawn Marsupenaeus japonicus. Mol. Immunol. 2006, 43, 2061-2069.

19. Rosa, R.D.; Stoco, P.H.; Barracco, M.A. Cloning and characterisation of cDNA sequences encoding for anti-lipopolysaccharide factors (ALFs) in Brazilian palaemonid and penaeid shrimps. Fish Shellfish Immunol. 2008, 25, 693-696.

20. Sharma, S.; Yedery, R.D.; Patgaonkar, M.S.; Selvaakumar, C.; Reddy, K.V. Antibacterial activity of a synthetic peptide that mimics the LPS binding domain of Indian mud crab, Scylla serrata anti-lipopolysaccharide factor (SsALF) also involved in the modulation of vaginal immune

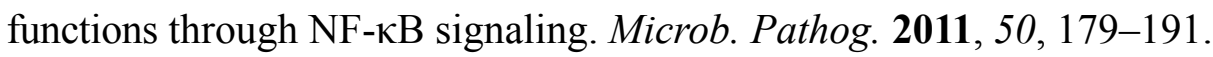

21. Pan, C.Y.; Chao, T.T.; Chen, J.C.; Chen, J.Y.; Liu, W.C.; Lin, C.H.; Kuo, C.M. Shrimp (Penaeus monodon) anti-lipopolysaccharide factor reduces the lethality of Pseudomonas aeruginosa sepsis in mice. Int. Immunopharmacol. 2007, 7, 687-700.

22. Guo, S.Y.; Li, S.H.; Li, F.H.; Zhang, X.J.; Xiang, J.H. Modification of a synthetic LPS-binding domain of anti-lipopolysaccharide factor from shrimp reveals strong structure-activity relationship in their antimicrobial characteristics. Dev. Comp. Immunol. 2014, 45, 227-232.

23. Li, S.H.; Guo, S.Y.; Li, F.H.; Xiang, J.H. Characterization and function analysis of an anti-lipopolysaccharide factor (ALF) from the Chinese shrimp Fenneropenaeus chinensis. Dev. Comp. Immunol. 2014, 46, 349-355.

24. Liu, Y.; Cui, Z.X.; Li, X.H.; Song, C.W.; Li, Q.Q.; Wang, S.Y. A new antilipopolysaccharide factor isoform (PtALF4) from the swimming crab Portunus trituberculatus exhibited structural and functional diversity of ALFs. Fish Shellfish Immunol. 2012, 32, 724-731.

25. Liu, Y.; Cui, Z.X.; Li, X.H.; Song, C.W.; Shi, G.H.; Wang, C.L. Molecular cloning, genomic structure and antimicrobial activity of PtALF7, a unique isoform of anti-lipopolysaccharide factor from the swimming crab Portunus trituberculatus. Fish Shellfish Immunol. 2013, 34, 652-659.

26. Li, S.H.; Zhang, X.J.; Sun, Z.; Li, F.H.; Xiang, J.H. Transcriptome analysis on Chinese shrimp Fenneropenaeus chinensis during WSSV acute infection. PLoS ONE 2013, 8, e58627. 
27. Liu, F.S.; Liu, Y.C.; Li, F.H.; Dong, B.; Xiang, J.H. Molecular cloning and expression profile of putative antilipopolysaccharide factor in Chinese shrimp (Fenneropenaeus chinensis). Mar. Biotechnol. 2005, 7, 600-608.

28. Yu, Y.; Xu, T.; Yu, Y.T.; Hao, P.; Li, X. Association of tissue lineage and gene expression: Conservatively and differentially expressed genes define common and special functions of tissues. BMC Bioinform. 2010, 11, S1; doi:10.1186/1471-2105-11-S11-S1.

29. Pongsomboon, S.; Wongpanya, R.; Tang, S.; Chalorsrikul, A.; Tassanakajon, A. Abundantly expressed transcripts in the lymphoid organ of the black tiger shrimp, Penaeus monodon, and their implication in immune function. Fish Shellfish Immunol. 2008, 25, 485-493.

30. Van De Braak, C.B.; Botterblom, M.H.A.; Taverne, N.; van Muiswinkel, W.B.; Rombout, J.; van der Knaap, W.P.W. The roles of haemocytes and the lymphoid organ in the clearance of injected Vibrio bacteria in Penaeus monodon shrimp. Fish Shellfish Immunol. 2002, 13, 293-309.

31. Hasson, K.W.; Lightner, D.V.; Mohney, L.L.; Redman, R.M.; White, B.M. Role of lymphoid organ spheroids in chronic Taura syndrome virus (TSV) infections in Penaeus vannamei. Dis. Aquat. Organ. 1999, 38, 93-105.

32. Johansson, M.; Keyser, P.; Sritunyalucksana, K.; Söderhäll, K. Crustacean haemocytes and haematopoiesis. Aquaculture 2000, 191, 45-52.

33. Okumura, T.; Nagai, F.; Yamamoto, S.; Yamano, K.; Oseko, N.; Inouye, K.; Oomura, H.; Sawada, H. Detection of white spot syndrome virus from stomach tissue homogenate of the kuruma shrimp (Penaeus japonicus) by reverse passive latex agglutination. J. Virol. Methods 2004, 119, 11-16.

34. Keller, R. Crustacean neuropeptides: Structures, functions and comparative aspects. Experientia 1992, 48, 439-448.

35. Liu, L.J.; Chen, Y.J.; Chang, Y.S.; Lee, C.Y. Neuroendocrine responses of a crustacean host to viral infection: Effects of infection of white spot syndrome virus on the expression and release of crustacean hyperglycemic hormone in the crayfish Procambarus clarkii. Comp. Biochem. Physiol. A Mol. Integr. Physiol. 2013, 164, 327-332.

36. Rodriguez, J.; Boulo, V.; Mialhe, E.; Bachere, E. Characterization of shrimp hemocytes and plasma components by monoclonal-antibodies. J. Cell Sci. 1995, 108, 1043-1050.

37. Sun, Y.M.; Li, F.H.; Xiang, J.H. Analysis on the dynamic changes of the amount of WSSV in Chinese shrimp Fenneropenaeus chinensis during infection. Aquaculture 2013, 376, 124-132.

38. Livak, K.J.; Schmittgen, T.D. Analysis of relative gene expression data using real-time quantitative PCR and the $2^{-\Delta \Delta C_{\mathrm{T}}}$ Method. Methods 2001, 25, 402-408.

39. Wiegand, I.; Hilpert, K.; Hancock, R.E.W. Agar and broth dilution methods to determine the minimal inhibitory concentration (MIC) of antimicrobial substances. Nat. Protoc. 2008, 3, 163-175.

(C) 2015 by the authors; licensee MDPI, Basel, Switzerland. This article is an open access article distributed under the terms and conditions of the Creative Commons Attribution license (http://creativecommons.org/licenses/by/4.0/). 